prospective or cross-sectional studies focussing on neuropsychiatric manifestations in SLE, defined according the ACR criteria of 1999. Study selection and data extraction was made in duplicate. We secured salient study characteristics, composition of cohorts, the definitions and the frequencies of neuropsychiatric manifestations. We assessed heterogeneity across reports and investigated sources of variation using meta-regression models.

Results The frequencies of severe manifestations found in the SSLE were $7.1 \%(49 / 688)$ for cerebrovascular events, $5.3 \%$ (37/688) for seizures and 6.5\% (45/688) for psychosis. The time-to-event analysis showed a linear relationship between duration of SLE and cumulative incidence of severe neuropsychiatric manifestations. Searches identified 530 studies and authors' contact yielded another unpublished report. We included 28 studies. The mean rates of the most commonly reported severe neuropsychiatric manifestations ranged in the magnitude of 50 percent points. Study characteristics and composition of cohorts could not explain heterogeneity of reported manifestation rates.

Conclusions The spectrum of neuropsychiatric manifestations in SLE is widely dispersed. The diagnostic work-up and the reporting of manifestations varied substantially across studies which may explain inconsistencies to some extent. We call for concerted efforts and a broad consensus regarding stringent definitions of neuropsychiatric SLE manifestations that allow targeted detection, particularly with view to timely intervention and patient outcomes.

\section{P142 LUPUS EUROPE - WHEN PATIENTS HELP RESEARCH}

${ }^{1,2}$ Alain Cornet, ${ }^{1}$ Jeanette Andersen, ${ }^{1}$ Kirsi Myllys, ${ }^{1}$ Anne Charlet, ${ }^{1}$ Annemarie Sluijmers, ${ }^{1}$ Helga Ovens. 'Lupus Europe, UK; ${ }^{2}$ CLAIR, Belgium

\subsection{6/lupus-2020-eurolupus. 185}

Background Patients involvement has massively changed over the years. From patients as 'objects' of care, we have moved to patient centric approaches and are now embarking at full speed in the era of patients as partners. To better meet this evolution, a radical change is taking place in Patient organisations. LUPUS EUROPE is one of the leading players. LUPUS EUROPE's 1st strategic objective is that 'People with Lupus participate, and benefit from, Lupus Research'.

Methods We have stepped up our capacity and capability by creating a Patient Advisory Network, organising 'naïve' patient panels - by and for lupus people; and running surveys in the community.

Results Our Patient Advisory Network now comprises 18 patients that are investing time and effort to develop their knowledge and to help academics, investigators, industry and other partners. They are currently engaged in more than 20 different projects. We are active in the ERN ReCONNET, EURORDIS and EJP RD. Our patient panels have already addressed the topics of: defining treatment; adherence; clinical trials and youth. Our latest research, on Hydroxychloroquine, obtained 3500 answers from all over Europe. Thanks to this stepped-up capability, we are also now ready to work with EMA, as an EMA eligible entity.

Conclusions Lupus Europe is ready to partner where it can add value, and to receive requests for support from researchers. In 2020 again, we will launch significant initiatives in the research area: A large scale survey on 'living with lupus in 2020'; the collection of feedback from participants in clinical trials to see how we can improve them and increase participation; and a further stepping up of the skills of our Patient Advisory Network. We need your help to increase our reach and work on jointly beneficial projects.

Acknowledgements Lupus Europe is mostly financed by contributions of industry - GSK, UCB, Idorsia, Boehringer, Janssen, Leo Pharma

\section{P143 LUPUS EUROPE - WHAT WE LEARNED FROM PATIENT PANELS}

${ }^{1,2}$ Alain Cornet, ${ }^{1}$ Jeanette Andersen, ${ }^{1}$ Kirsi Myllys. ' Lupus Europe, UK; ${ }^{2}$ CLAIR, Belgium

\subsection{6/lupus-2020-eurolupus. 186}

Background Much remains to be discovered about Lupus. Not just disease mechanisms and new treatments, but also better understanding the day to day issues faced by patients and their relatives, the impact on social and psychological functioning,... To help people living with lupus bring their life experience to the table, LUPUS EUROPE created patient panels, by and for people with lupus.

Methods Over the past years, Lupus Europe conducted 3 patient panels. Each panel, from Friday evening to Sunday afternoon brought together 10 to 12 diverse European (semi-) naïve patients around 1 theme, with $8-10$ 'activities' exploring the subject. The panels were moderated by people living with lupus themselves.

Results Our panels have brought insights that we think are relevant for doctors and researchers in lupus. Amongst those:

a. Patients define treatment much broader than doctors. They view treatment as 'any product or activity that aims at improving the person with lupus' quality of life', not just prescription drugs.

b. The patient-doctor relationship impacts adherence, with unmet needs in the area of 'understanding each component of the treatment', 'having concerns raise acknowledged even if not irrelevant to diagnose' and 'feeling ownership for the treatment plan'.

c. Youth acknowledge their top issue is taking pills every day, but preferred to focus on collective issues. Young women's feeling of guilt (imposing limits, having children, 'contaminating' them...) is likely underestimated by doctors and patient organizations.

d. Young people are more positive on their life than average patients. They perceive lupus as being 'all over their lives', but want to make sure they are not ruled by it, and want to have no obligations relating to it...

Many additional panel conclusions are available on https:// www.lupus-europe.org/patient-panels/

Conclusions Lupus Europe's panels provide an opportunity to gather insights that can feed the patient-doctor relationship and contribute to a better understanding of the disease.

Acknowledgements Lupus Europe is mostly financed by contributions of industry - GSK, UCB, Idorsia, Boehringer, Janssen, Leo Pharma 